\title{
NFIB promotes cell growth, aggressiveness, metastasis and EMT of gastric cancer through the Akt/Stat3 signaling pathway
}

\author{
CHUANQING WU*, XIAOJIE ZHU*, WEIZHEN LIU, TUO RUAN, WENZE WAN and KAIXIONG TAO \\ Department of Gastrointestinal Surgery, Union Hospital, Tongji Medical College, \\ Huazhong University of Science and Technology, Wuhan 430022, China
}

Received January 16, 2018; Accepted May 31, 2018

DOI: 10.3892/or.2018.6574

\begin{abstract}
Nuclear factor I/B (NFIB) plays a crucial role in the progression of several types of cancers. However, its role in gastric cancer (GC) remains unclear. The present study revealed that NFIB was highly expressed in GC tissues and was positively associated with the clinicopathological features of GC patients. Downregulation of NFIB inhibited the tumor growth, migration and aggression of MKN45 and HGC27 cells in vitro. In addition, NFIB expression promoted epithelial-mesenchymal transition (EMT), which was accompanied with decreased E-cadherin and increased vimentin expression. Since AKT and Stat3 play an important role in EMT and tumor progression, we examined whether there is a correlation between NFIB and AKT/Stat 3 signaling pathways in GC. Our results revealed that NFIB exhibits its oncogenic functions in GC development by regulating the phosphorylation of both AKT and Stat 3 molecules. Knocking down the NFIB expression may enhance the phosphorylation of AKT while inhibiting the Stat3 phosphorylation, suggesting that the AKT/Stat 3 signaling pathway may be the downstream target of NFIB with which it exerts its roles on GC development. These results revealed that NFIB promotes tumor growth and aggressiveness of GC. In addition, downregulation of NFIB alters the protein kinase $\mathrm{B} /$ signal transducers and activators of transcription 3 (AKT/Stat3) axis, which could be a potential molecular mechanism for precise target treatment of GC.
\end{abstract}

\section{Introduction}

According to the latest statistics, gastric cancer (GC) remains to be the third most common malignant disease globally,

Correspondence to: Dr Kaixiong Tao, Department of Gastrointestinal Surgery, Union Hospital, Tongji Medical College, Huazhong University of Science and Technology, 1277 Jiefang Avenue, Wuhan 430022, China

E-mail: kaixiongtao@hust.edu.cn

*Contributed equally

Key words: NFIB, Akt, Stat3, gastric cancer, molecular therapy and its lethal malignant nature is responsible for the high mortality and morbidity of patients (1). In China, statistical data confirmed nearly 1 million GC cases and cancer-related deaths, making it the second most lethal malignancy in the Chinese population (2). Although multiple factors contribute to the morbidity and mortality of GC, tumor growth and metastasis are key sources to the malignancy of GC (3). Methods that could alleviate or reverse them are expected in the targeted treatment of GC and thus are worthy of further investigation.

The PI3K/AKT/Stat3 signaling pathway is a classic, multi-functional mechanism which is involved with the development and progression of numerous cancers, such as human bladder, breast and colorectal cancer (4-6). It has been thoroughly studied and is believed to contribute to the malignancy of GC as well, particularly to its metastatic characteristics (7).

Nuclear factors and their receptors are indispensable in the development of tumor growth, metastasis and epithelial-mesenchymal transition (EMT) in several types of cancers (8). Nuclear factor I/B (NFIB) is a recently discovered member of the nuclear factor family (which includes NFI-A, $\mathrm{B}$ and $\mathrm{X}$ ) and emerging evidence has shown that NFIB, besides functioning during normal somatic development, is also involved in the generation and progression of various types of cancer, including small-cell lung cancer, melanoma and breast cancer, through different molecular mechanisms such as forming the oncogenic NFIB-MYB gene fusion or increasing the chromatin accessibilities of its downstream targets (9-11). Nevertheless, research on the functions and involvement of NFIB on the development of GC has not been reported yet.

In the present study, we focused on the roles played by NFIB on the malignant behavior of GC cells. Our research demonstrated that NFIB is an oncogenic molecule which contributes to the malignancy of GC by enhancing the proliferation, migration, aggression and EMT processes of GC cells. We further discovered that NFIB exerts its function by regulating CXCR4, AKT and Jak2/Stat3 pathway members. Silencing of NFIB expression enhanced AKT phosphorylation, in addition to the downregulation of CXCR4 expression as well as the phosphorylation of Jak $2 /$ Stat 3 pathway members. Our study for the first time revealed NFIB as a developmental contributor of GC and a potential target for the molecular treatment of GC. 


\section{Materials and methods}

Quantitative real-time polymerase chain reaction. Total RNA was extracted from tissues and cultured cells by TRIzol reagent (Takara Biotechnology Co., Ltd., Dalian, China). The quantity of RNA was assessed using an ultraviolet spectrophotometer at absorbances of 260 and $280 \mathrm{~nm}$. The ratio of optical density (OD) 260/280 between 1.7 and 2.1 suggested an adequate purity for subsequent experiments. The primers for evaluating the NFIB expression in GC tissues and cell lines was: i) NFIB forward, 5'-AAAAAGCATGAGAAG CGAATGTC-3' and reverse, 5'-ACTCCTGGCGAATATCTT TGC-3'; ii) GAPDH forward, 5'-ACAACTTTGGTATCGTGG AAGG-3' and reverse, 5'-GCCATCACGCCACAGTTTC-3'. The reverse transcription reactions were carried out using the PrimeScript $^{\mathrm{TM}}$ One Step qPCR kit (Takara Biotechnology). The messenger RNA (mRNA) expression analysis of NFIB was performed by real-time polymerase chain reaction (qPCR) using a standard SYBR-Green PCR kit (Takara Biotechnology) protocol on an Applied Biosystems 7500 Real-Time PCR system (Applied Biosystems; Thermo Fisher Scientific, Inc., Waltham, MA, USA) according to the manufacturer's instructions. The reaction conditions of qPCR were: $95^{\circ} \mathrm{C}$ for $30 \mathrm{sec}$, $60^{\circ} \mathrm{C}$ for $30 \mathrm{sec}, 40 \mathrm{cycles}$ at $95^{\circ} \mathrm{C}$ for $30 \mathrm{sec}, 60^{\circ} \mathrm{C}$ for $30 \mathrm{sec}$, and $72^{\circ} \mathrm{C}$ for $30 \mathrm{sec}$. Glyceraldehyde 3-phosphate dehydrogenase (GAPDH) expression was regarded as the endogenous control in each sample. The relative gene expression levels were calculated using the comparative $\mathrm{Cq}(\Delta \Delta \mathrm{Cq})$ method, in which the relative expression is calculated as $2-\Delta \Delta \mathrm{Cq}$, and $\mathrm{Cq}$ represents the threshold cycle (12). Each experiment was conducted in triplicate.

Cell procurement and maintenance. The normal human gastric normal epithelium cell line GES1 and the GC cell lines AGS, MKN45, HGC27, MGC803 and SGC7901 were preserved and obtained from the Laboratory of General Surgery, Union Hospital, Tongji Medical College. All cell lines were cultured in the cell culture medium RPMI-1640 (HyClone; GE Healthcare Life Sciences, Logan, UT, USA) mixed with $10 \%$ fetal bovine serum (FBS; ScienCell Research Laboratories, Inc., San Diego, CA, USA), streptomycin (100 $\mu \mathrm{g} / \mathrm{ml})$ and penicillin (100 U/ml; both from Sigma-Aldrich; Merck, St. Louis, MO, USA) at $37^{\circ} \mathrm{C}$ and $5 \% \mathrm{CO}_{2}$. Antibodies against NFIB (dilution 1:500; cat. no. ab186738) and GAPDH (dilution 1:1,000; cat. no. ab9485) were purchased from Abcam (Cambridge, MA, USA), while E-cadherin (dilution 1:1,000; cat. no. sc-71009), $\mathrm{N}$-cadherin (dilution 1:1,000; cat. no. sc-59987) and vimentin (dilution 1:1,000; cat. no. sc-80975) were purchased from Santa Cruz Biotechnology, Inc. (Dallas, TX, USA). Akt/p-Akt (dilution 1:1,000; cat. no. 9272S/4060S) and Stat3/p-Stat3 (dilution 1:1,000; cat. no. $12640 \mathrm{~S} / 9145 \mathrm{~S}$ ) antibodies were purchased from Cell Signaling Technology, Inc. (Danvers, MA, USA).

Cell viability assay. HGC27 and MKN45 cells in logarithmic growth were washed twice with phosphate-buffered saline (PBS) and seeded on a 96-well plate with 6 duplicate wells at a final density of $5 \times 10^{3}$ cells/well at $37^{\circ} \mathrm{C}$ for $24 \mathrm{~h}$. Then, the cells were transfected with either NFIB siRNAs or NFIB controls under the same concentration of $100 \mu \mathrm{M}$.
At indicated time-points, $20 \mu \mathrm{l}$ of $5 \mathrm{mg} / \mathrm{ml} \mathrm{3-(4,5-dimeth-}$ ylthiazol-2-yl)-2,5-diphenyltetrazolium bromide (MTT; Sigma-Aldrich; Merck KGaA) was added into each well and incubated for another $4 \mathrm{~h}$ at $37^{\circ} \mathrm{C}$ and $5 \% \mathrm{CO}_{2}$. Then, the culture medium was removed and $150 \mu 1$ dimethyl sulfoxide (DMSO) was added to each MTT-treated well for $10 \mathrm{~min}$ to dissolve the crystals. The absorbance at $490 \mathrm{~nm}$ was determined using a microplate reader (Molecular Devices, LLC, Sunnyvale, CA, USA) to assess cell viability. The experiment was repeated at least 3 times.

Colony formation assay. Transfected or non-transfected HGC27 and MKN45 cells (500 cells/well) were evenly seeded on 6-well plates in triplicate and were grown for $48 \mathrm{~h}$ in RPMI-1640 medium with $10 \% \mathrm{FBS}$ at $37^{\circ} \mathrm{C}$. Then, both cell types were divided into 2 groups and transfected with either NFIB siRNAs or NFIB controls under the same concentration of $100 \mu \mathrm{M}$, separately. After 14 days, the cells were fixed with $4 \%$ paraformaldehyde (Sigma-Aldrich; Merck) for $30 \mathrm{~min}$, stained with Giemsa (Sigma-Aldrich; Merck) for $20 \mathrm{~min}$, and photographed under an inverted fluorescence microscope (IX51; Olympus Corp., Tokyo, Japan) equipped with an Olympus Qcolor 3 digital camera (Olympus Corp.). The number of colonies (>50 cells) were analyzed using an inverted microscope (Nikon, Inc., Garden City, NY, USA).

Cell migration and invasion assay. Cell migration and invasion were assessed with Transwell chambers (Corning Incorporated, Corning, NY, USA) containing 24-well inserts with $8-\mu \mathrm{m}$ pores in the presence or absence of Matrigel ECM (BD Biosciences, Franklin Lakes, NJ, USA), according to the manufacturer's protocols. After $48 \mathrm{~h}$ of transfection, both MKN45 (100,000/chamber) and HGC27 (100,000/chamber) cells were seeded to the upper chamber filled with $\mathrm{Gibco}^{\mathrm{TM}}$ Opti-MEM $^{\text {тм }}$ (Thermo Fisher Scientific, Inc.) serum-free medium and incubated for an additional $24 \mathrm{~h}$ for migration or $48 \mathrm{~h}$ for invasion. The lower chamber was filled with $600 \mu \mathrm{l}$ Gibco $^{\text {TM }}$ RMPI-1640 medium (Thermo Fisher Scientific, Inc.) mixed with $10 \%$ FBS (ScienCell Research Laboratories, Inc., Carlsbad, CA, USA). Then, the cells in the upper chamber were wiped off, and the penetrated cells were fixed in $4 \%$ paraformaldehyde and stained with crystal violet staining solution (dilution rate: 1:3) at room temperature for $15 \mathrm{~min}$. Cells were quantified by counting in 5 randomly selected fields for each membrane under light microscope with an x100 magnification, and the average number of cells from 3 independent tests was regarded as the migration or invasion value.

Western blot analysis. The total proteins from treated cells and biopsy samples of gastric tumors were extracted with radioimmunoprecipitation assay (RIPA) lysis buffer (Pierce; Thermo Fisher Scientific, Inc., Waltham, MA, USA). The contents of the extracted proteins were quantified using the BCA Protein Assay Reagent kit (Pierce; Thermo Fisher Scientific, Inc.). For analysis of protein expression, equal amounts of protein $(40 \mu \mathrm{g})$ from each sample were run on $12 \%$ sodium dodecyl sulfate-polyacrylamide gel electrophoresis (SDS-PAGE) and electro-transferred to polyvinylidene difluoride (PVDF) membranes (EMD Millipore, Billerica, MA, USA). Following blocking with $5 \%$ bovine serum albumin for $2 \mathrm{~h}$ at $37^{\circ} \mathrm{C}$, the 
membranes were probed with the primary antibody against phosphorylated Akt (cat. no. 4060S), Akt (cat. no. 9272S), phosphorylated Stat3 (cat. no. 9145S), Stat3 (cat. no. 12640S) and GAPDH (cat. no. ab9485) at a concentration of 1:1,000 overnight at $4^{\circ} \mathrm{C}$. After being washed 3 times with Tris-buffered saline with Tween-20 (TBST) buffer, the horseradish peroxidase-goat anti-rabbit antibodies (cat. no. 10285-1-AP; ProteinTech, Co., Wuhan, China) were added at 1:3,000 dilutions and incubated with the membranes at room temperature for $1 \mathrm{~h}$. After being washed again, the protein bands were visualized and quantified using Electrochemiluminescence Plus Detection system (EMD Millipore). GAPDH was used as an internal reference.

Immunohistochemistry (IHC) analysis. Immunohistochemistry was performed with polymer-based technology (EnVision; Dako; Agilent Technologies, Inc., Santa Clara, CA, USA) as described by previous studies (13). The study was approved by the Ethics Committee of Union Hospital, Tongji Medical College, Huazhong University of Science and Technology. The gathering and use of the patient data and tissues were consented by all patients involved. The tissue specimens of GC patients and adjacent normal tissues (male:female, 2:17; age range, 37-83 years) collected by the Department of Gastrointestinal Surgery of Union Hospital from November 2013 to December 2016 were fixed with 4\% paraformaldehyde overnight at $4^{\circ} \mathrm{C}$ and then embedded in paraffin, sliced into 4-mm sections with a microtome and then fixed onto slides. The tissue sections were dewaxed with xylene and rehydrated with graded alcohol concentrations as instructed by the standard procedures. The sections were then treated in EDTA (pH 8.0) and autoclaved at $121^{\circ} \mathrm{C}$ for $5 \mathrm{~min}$ to retrieve the antigenicity. After being washed in PBS (0.1 M, pH 7.4, 3 times for $5 \mathrm{~min}$ ), the endogenous peroxidase was blocked with $3 \%$ hydrogen peroxide for $15 \mathrm{~min}$ at room temperature. After being washed again with PBS, the slides were incubated with primary antibodies for $1 \mathrm{~h}$ at room temperature. The primary antibody and dilution were: NFIB (cat. no. ab186738; Abcam), 1:100 dilution. Immunostaining was performed using the EnVision system with diaminobenzidine (Dako Cytomation, Glostrup, Denmark). The relative intensity of NFIB expression was evaluated with the Image-Pro Plus (IPP version 6.0; Media Cybernetics, Inc., Silver Spring, MD, USA).

Statistical analysis. Statistical analyses were performed using SPSS 20.0 software package (SPSS, Inc., Chicago, IL, USA). All data were compared and analyzed as the mean \pm standard deviation (SD) from at least 3 independent experiments. Statistical significance was estimated with Student's t-test between 2 groups and one-way analysis of variance (ANOVA) followed by the least significant difference method for 3 and more groups. The association between NFBI expression and clinical parameters was analyzed using a Chi-squared test. $\mathrm{P}<0.05$ was considered to indicate a statistically significant difference.

\section{Results}

NFIB is highly expressed in GC tissues and cell lines. For starters, we explored the functions of NFIB in GC by examining
Table I. Clinicopathological characteristics of patients

\begin{tabular}{|c|c|c|c|c|}
\hline \multirow[b]{2}{*}{ Clinicopathological factors } & \multirow[b]{2}{*}{$\mathrm{n}$} & \multicolumn{2}{|c|}{$\begin{array}{c}\text { NFIB } \\
\text { expression }\end{array}$} & \multirow[b]{2}{*}{ P-value } \\
\hline & & High & Low & \\
\hline Age (years) & & & & 0.5766 \\
\hline$>60$ & 7 & 5 & 2 & \\
\hline$<60$ & 30 & 19 & 11 & \\
\hline Sex & & & & 0.1881 \\
\hline Male & 20 & 15 & 5 & \\
\hline Female & 17 & 9 & 8 & \\
\hline Pathological T stage & & & & $0.0011^{\mathrm{a}}$ \\
\hline $\mathrm{T} 1+\mathrm{T} 2$ & 10 & 2 & 8 & \\
\hline $\mathrm{T} 3+\mathrm{T} 4$ & 27 & 22 & 5 & \\
\hline Pathological N stage & & & & $0.0353^{\mathrm{a}}$ \\
\hline No & 22 & 11 & 11 & \\
\hline $\mathrm{N} 1+\mathrm{N} 2$ & 15 & 13 & 2 & \\
\hline Tumor differentiation & & & & $0.0043^{\mathrm{a}}$ \\
\hline High & 5 & 0 & 5 & \\
\hline Moderate & 26 & 19 & 7 & \\
\hline Low & 6 & 5 & 1 & \\
\hline
\end{tabular}

The NFIB staining intensity was independently scored as follows: $<50 \%$ staining was identified as low expression, and $>50 \%$ as high expression. ${ }^{\mathrm{a}} \mathrm{P}<0.05$. Chi-squared test was used for the evaluation of statistical significance. NFIB, nuclear factor I/B.

its expression levels in GC tissues as well as GC cell lines. NFIB expression was examined in 37 primary GC tissues coupled with their corresponding normal adjacent mucosa, as well as the human GC (AGS, MKN45, HGC27, MGC803 and SGC7901) cell lines. A strong and widely-distributed cytonuclear staining pattern of NFIB was detected in GC specimens by immunohistochemical (IHC) staining, whereas the corresponding normal mucosa exhibited weak or no staining of NFIB (Fig. 1A and B). Western blotting, as shown in Fig. 1C, further confirmed that the expression of NFIB was significantly higher in GC tissues than the corresponding adjacent tissues. In addition, western blotting revealed that the expression level of NFIB was also elevated in most human GC cell lines, among which the MKN45 and HGC27 cell lines had the highest expression (Fig. 1D). Consistent with the NFIB expression comparisons at the protein levels, the mRNA levels of NFIB in GC tissues were markedly enhanced, compared with those in adjacent non-cancerous tissues (Fig. 1E).

Elevated NFIB expression is associated with poorer clinical prognosis of GC patients. To investigate the roles played by NFIB in the clinical characteristics as well as the prognosis of GC patients, we compared the relationships between the expression of NFIB in GC tissues and the clinical features of 37 GC patients that we collected (Table I).

Statistical analysis revealed that NFIB overexpression in GC patients was significantly associated with more advanced 
A
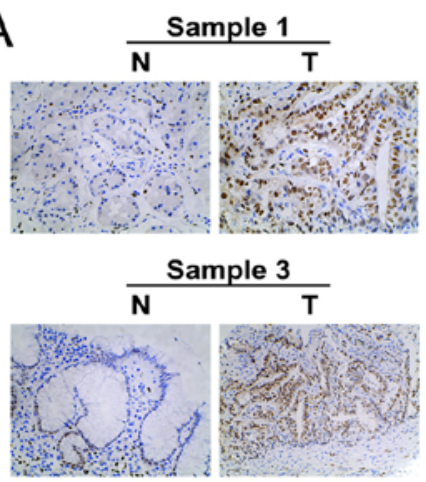
Sample 3

Sample 2

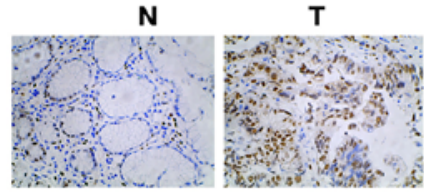

Sample 4

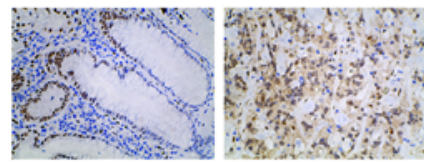

C

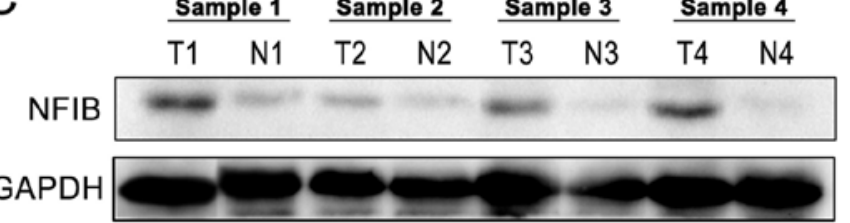

$\mathrm{D}$

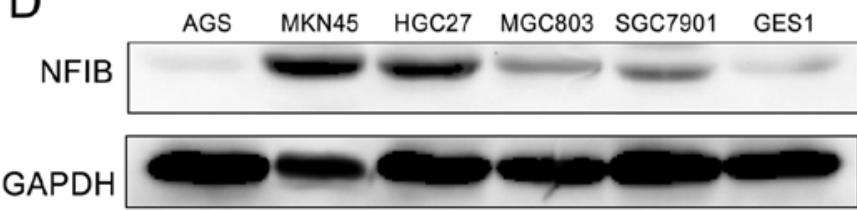

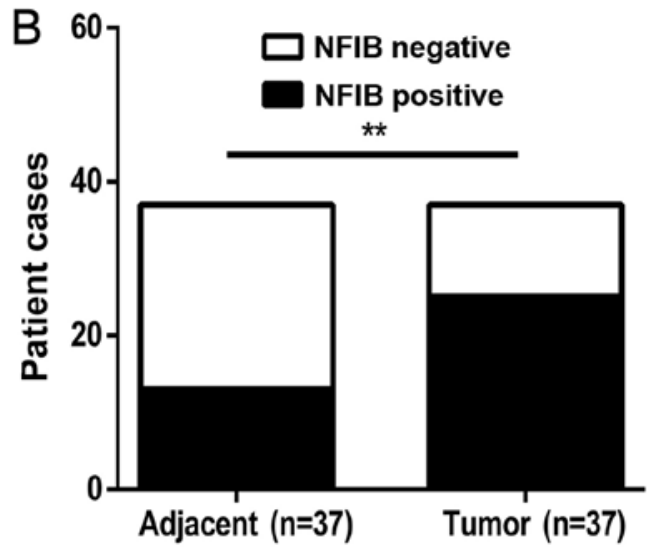

Patient groups

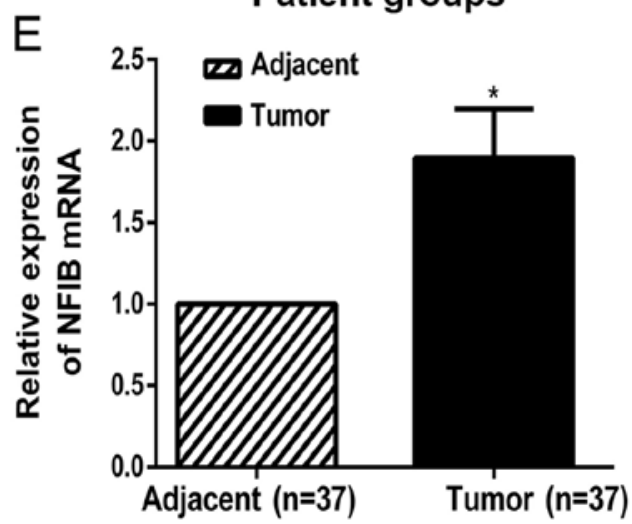

Figure 1. NFIB is highly expressed in gastric cancer (GC) tissues and cell lines. (A) The immunohistochemical (IHC) staining detected strong NFIB expression in the cytonuclei of GC tumor tissues, whereas in adjacent non-cancerous tissues, the expression of NFIB was weak and majorly restricted to the gland area. (B) The IHC detection of the mRNA samples of $37 \mathrm{GC}$ tissue pairs revealed an elevated NFIB expression ratio (64.8\% positive, 24/37) in GC tissues, compared to the lower expression ratio of NFIB (32.4\% positive, 12/37) in adjacent non-cancerous tissues. (C) Western blotting revealed a higher protein expression of NFIB in GC tissue samples compared to the adjacent normal tissues. N, normal tissue; T, GC tissue. (D) NFIB expression was examined by western blotting in 5 human GC cell lines (AGS, MKN45, HGC27, MGC823 and SGC7901) and the normal human gastric mucosal cell line GES1. Higher NFIB expression was detected in all human GC cell lines except for AGS, compared with the GES1 cell line. (E) Consistent with the IHC and western blot analysis findings, the RT-PCR detection of the mRNA samples of the 37 GC tissue pairs confirmed significantly higher NFIB expression in GC tissues (1.7-fold relative to the adjacent non-cancerous tissues). ${ }^{*} \mathrm{P}<0.05,{ }^{* *} \mathrm{P}<0.01$. NFIB, nuclear factor I/B.

tumor-node-metastasis (TNM) stages and tumor differentiation $(\mathrm{P}<0.05)$. Other clinical parameters, including age and sex, were not statistically significantly associated to NFIB overexpression. These statistical analysis data predicted NFIB as an oncogenic molecule in GC and revealed it to be associated with differentiation and metastasis, which prompted us to further study its roles and functions with respect to the aggressiveness, invasion, metastasis and proliferation of GC cells.

Elevated NFIB expression promotes the migratory and invasive characteristics of GC cell lines. Since our data demonstrated that NFIB expression is significantly elevated in GC tissue samples and cell lines, as well as associated to poorer clinical prognosis, we next aimed to find out whether NFIB overexpression, to some extent, is responsible for the malignancy of GC. We chose the two cell lines, MKN45 and HGC27, as the cell tools for our further research, since they expressed the highest levels of NFIB protein among all the examined GC cell lines.

To investigate the effects of NFIB silencing on the migration and invasion aspects of GC cells, we first examined the efficacy of NFIB-siRNA on decreasing NFIB expression.
Both MKN45 and HGC27 cell lines were divided into two subgroups and designated as siNFIB and siNC groups, which were transfected with either the NFIB-siRNA (siNFIB) or the negative-control siRNA (siNC), respectively. The western blotting revealed that siNFIB significantly decreased the expression of NFIB in both cell lines, compared with the siNC groups (Fig. 2).

We further investigated the effects of NFIB knockdown on the migratory and invasive capacities of the GS cell lines with Transwell assays. The results revealed that the downregulation of NFIB markedly inhibited the migration (Fig. 3C) as well as the invasion (Fig. 3B) abilities of GC cells. When transfected with NFIB-siRNA, both MKN45 (Fig. 3A) and HGC27 (Fig. 3B) cells became less effective on penetrating the Transwell chamber membranes and extracellular matrix (ECM) coatings.

NFIB expression promotes the colony formation in GC cell lines. Since the tumorigenicity of GC cells is one of the most important factors for cancer malignancy, we also explored the roles of NFIB in the tumorigenicity properties of GC with colony formation assays. As shown in Fig. 4, the siNFIB groups 
A
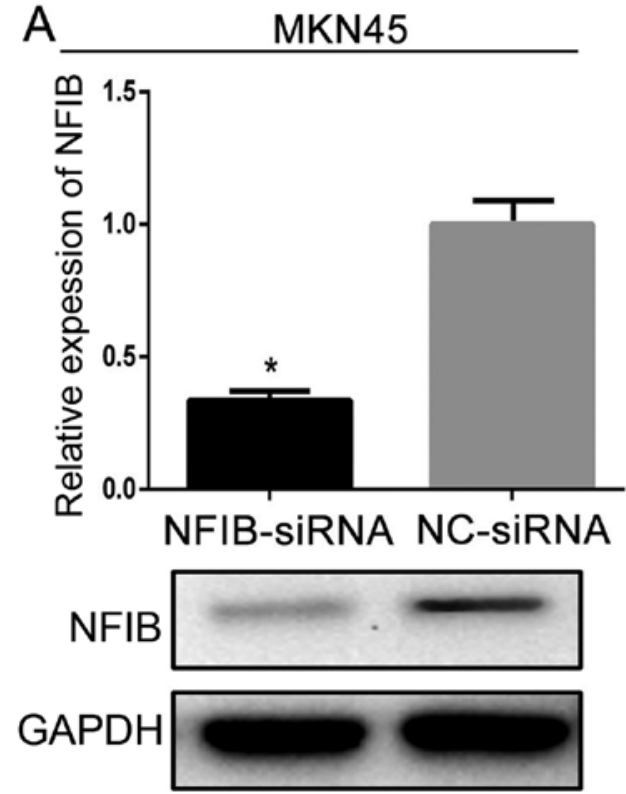

B

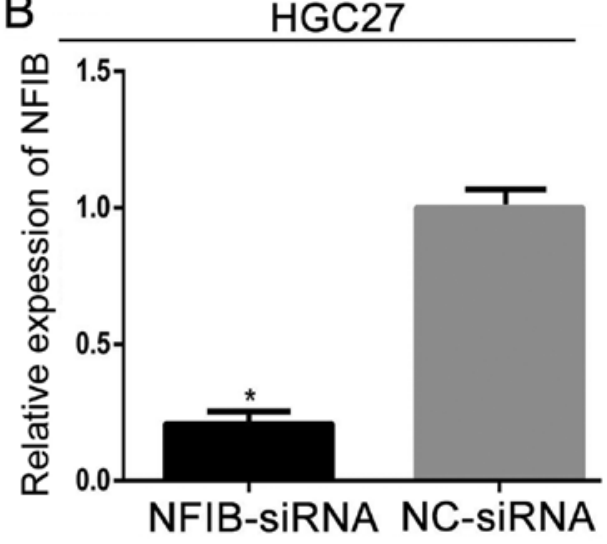

NFIB

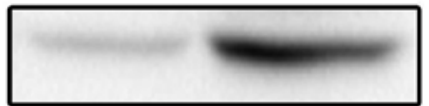

GAPDH

Figure 2. NFIB-siRNA is transfected to gastric cancer (GC) cells and knockdown efficacy is examined by western blot analysis. The results revealed that NFIB-siRNA was efficient at knocking down the expression of NFIB in both (A) MKN45 and (B) HGC27 cell lines. When the cells were treated with NFIB-siRNA transfection, the expression of NFIB was significantly suppressed at the protein level ("P<0.05). NFIB, nuclear factor I/B.

A

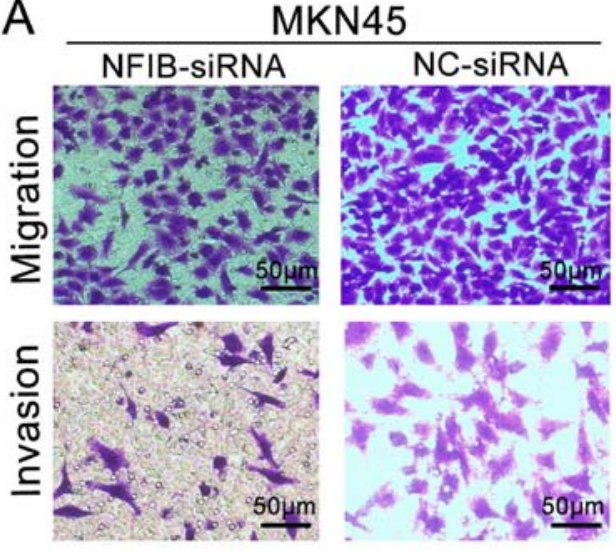

C

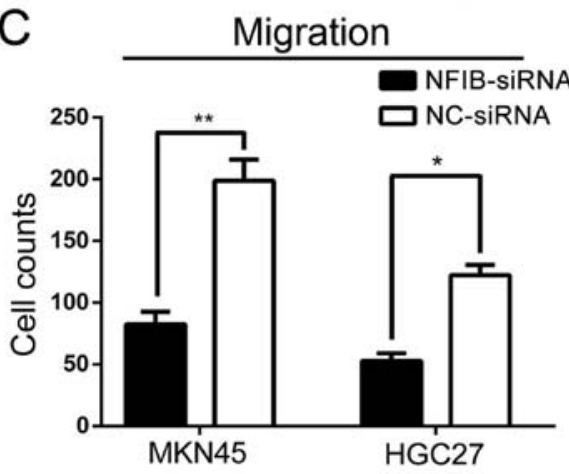

B

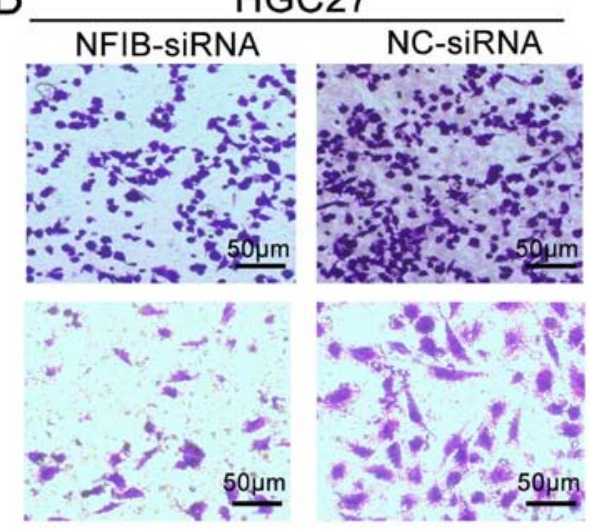

D

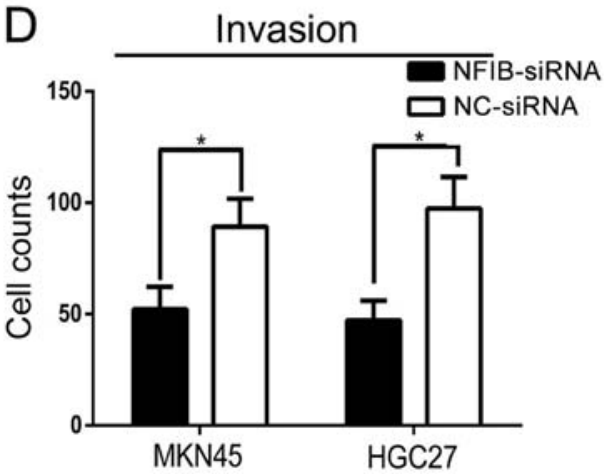

Figure 3. Migration and invasion capabilities of gastric cancer (GC) cells are determined by 3 independent Transwell assays. The results of the Transwell assays revealed that after knocking down the expression of NFIB, the number of cells which penetrated the chamber membranes as well as the ECM matrix of both (A) MKN45 and (B) HGC27 were decreased. (C and D) Statistical analysis revealed that compared with the NC-siRNA groups, the NFIB-siRNA groups had significantly fewer numbers of penetrated cells (the analysis was based on 3 independent trials; ${ }^{*} \mathrm{P}<0.05,{ }^{* *} \mathrm{P}<0.05$ ). NFIB, nuclear factor I/B.

of HGC27 (Fig. 4A) and MKN45 (Fig. 4C) displayed inhibited ability to form colonies. The numbers and sizes of the colonies were significantly limited for both cell lines compared with the NC groups (Fig. 4B and D).
NFIB expression promotes the proliferation and viability of $G C$ cell lines. Another important factor of malignancy for $\mathrm{GC}$ is the proliferation of GC cells. We studied the contribution of NFIB on the proliferation and viability aspects of 
A

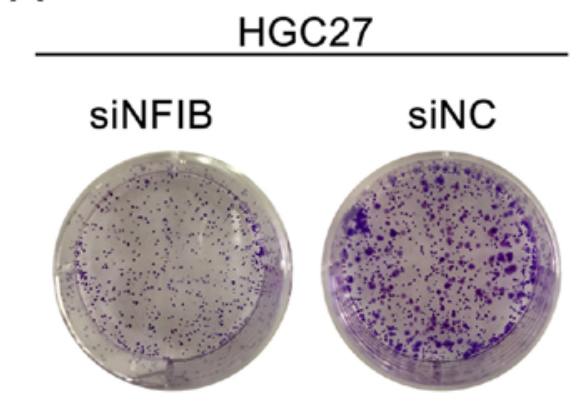

C

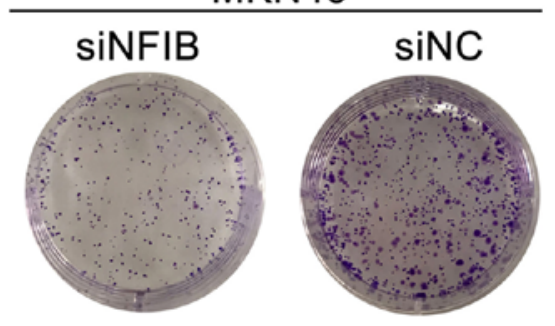

$\mathrm{B}$
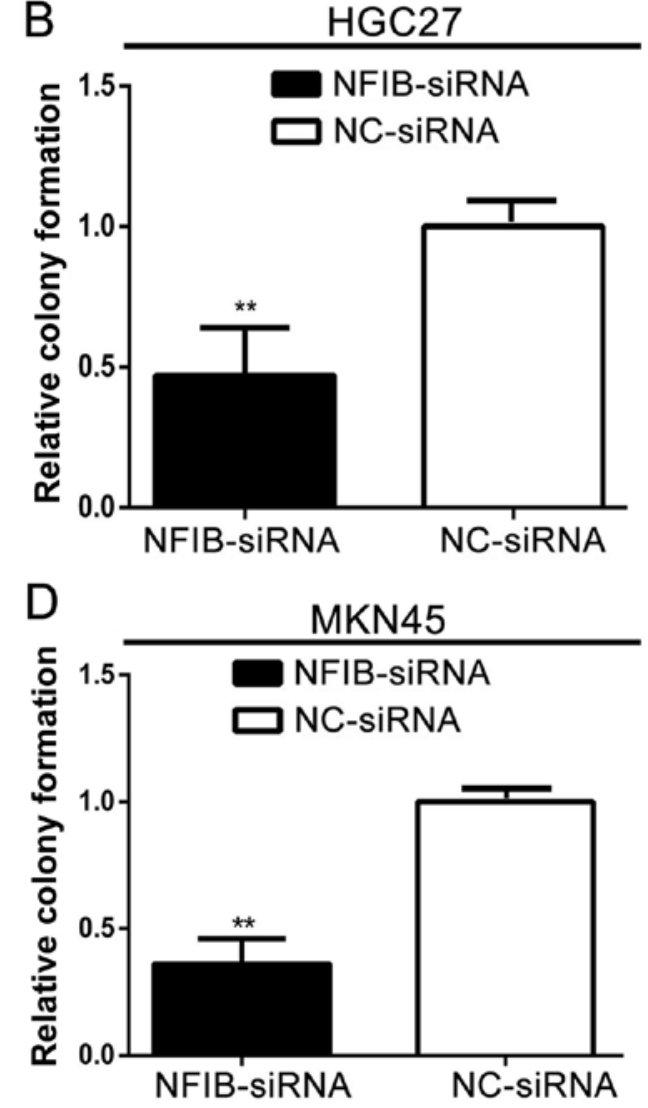

Figure 4. The tumorigenicity of gastric cancer (GC) cells was examined with semi-quantified methods by colony formation assays. The ability to form colonies of (A) HGC27 and (C) MKN45 cells in the siNFIB groups was distinctly inhibited. (B and D) Statistical comparison further confirmed the decreased tumorigenicity of the siNFIB groups (the analysis was based on 3 independent trials; ${ }^{* *} \mathrm{P}<0.01$ ). NFIB, nuclear factor I/B; siNFIB, NFIB-siRNA; siNC, NC-siRNA.
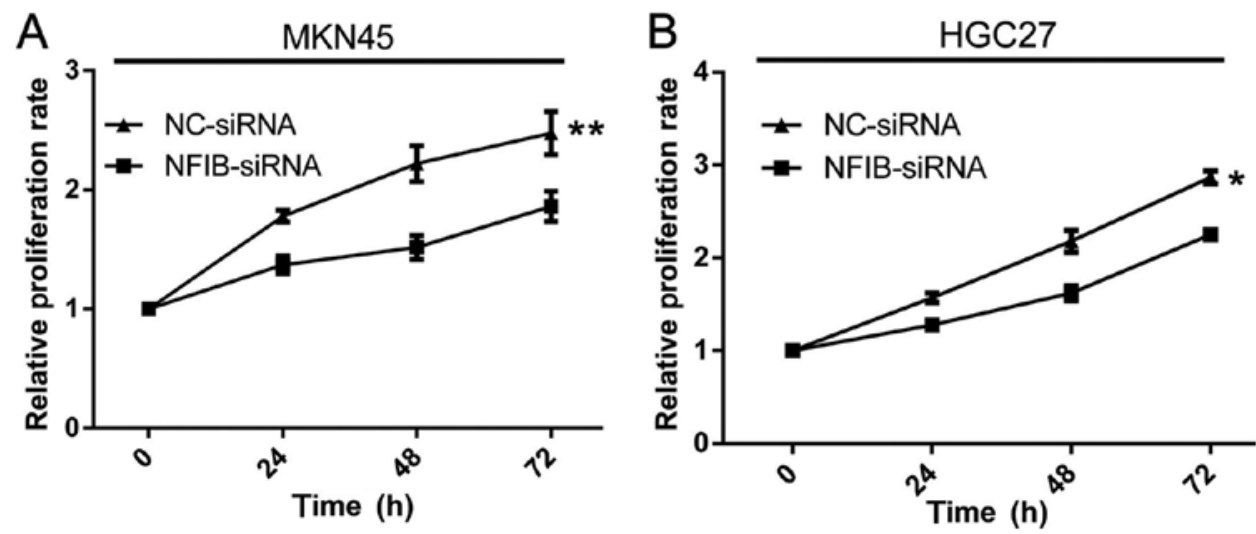

Figure 5. The proliferation rates of (A) MKN45 and (B) HGC27 cells were examined by MTT assay 2 days after transfection with NFIB-siRNA or NC-siRNA. The OD values reflected the number of cells. Statistical analysis was based on 3 independent trials with 5 -wells for each group $\left({ }^{*} \mathrm{P}<0.05,{ }^{* *} \mathrm{P}<0.01\right)$. NFIB, nuclear factor I/B; GC, gastric cancer; NC, negative control; OD, optical density.

GC cells by MTT assays. Relative to the higher proliferation ability of the siNC groups, the proliferation rates of the siNFIB groups of MKN45 (Fig. 5A) and HGC27 (Fig. 5B) cells were significantly reduced. The inhibition effects of NFIB knockdown on the proliferation of GC cells were most evident during the first $48 \mathrm{~h}$. Notably, after $48 \mathrm{~h}$, the siNFIB groups had almost restored the proliferation viabilities. This may be explained by the effective duration of the NFIB-siRNA which may have expired.
NFIB expression promotes the EMT of GC cell lines. EMT has been studied and is believed to contribute to the migration, transformation, invasion and malignant behavior of numerous types of cancer, including GC. We thus investigated the associations between NFIB expression and the EMT properties of GC cells. The western blot results (Fig. 6) revealed that compared with the siNC groups, the siNFIB groups of HGC27 and MKN45 cells expressed a higher level of E-cadherin and lower levels of $\mathrm{N}$-cadherin and vimentin, suggesting that knockdown 
A

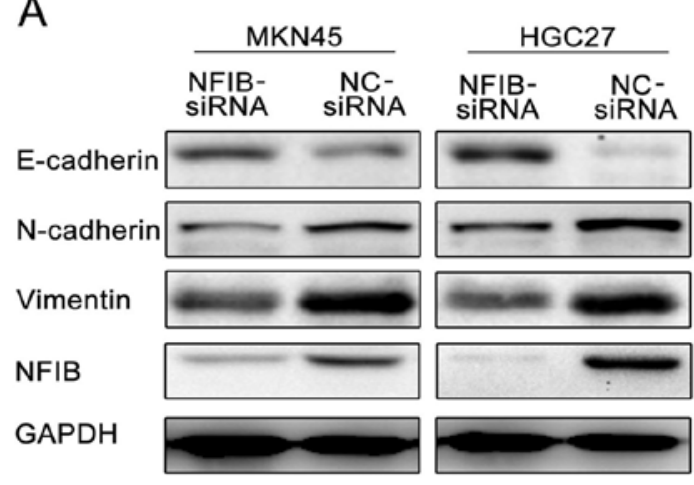

$\mathrm{B}$

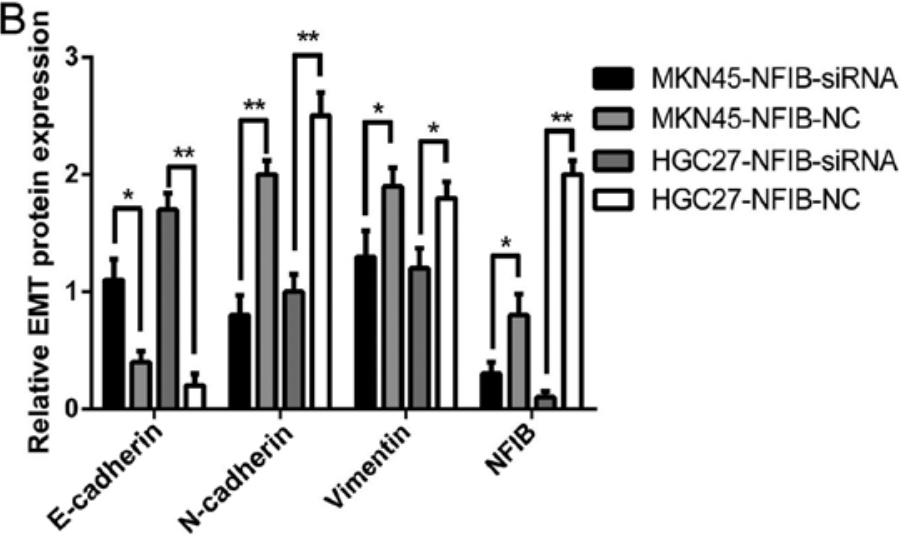

Figure 6. Western blot analysis results of EMT proteins in MKN45 and HGC27 cell lines. The expression levels of E-cadherin were elevated following the knockdown of NFIB in both NFIB-siRNA groups, while the expression of N-cadherin and vimentin was concurrently downregulated, which was in accordance to the predisposition of EMT transformation ( $\left.{ }^{*} \mathrm{P}<0.05,{ }^{* *} \mathrm{P}<0.01\right)$. NFIB, nuclear factor I/B; EMT, epithelial-mesenchymal transition.
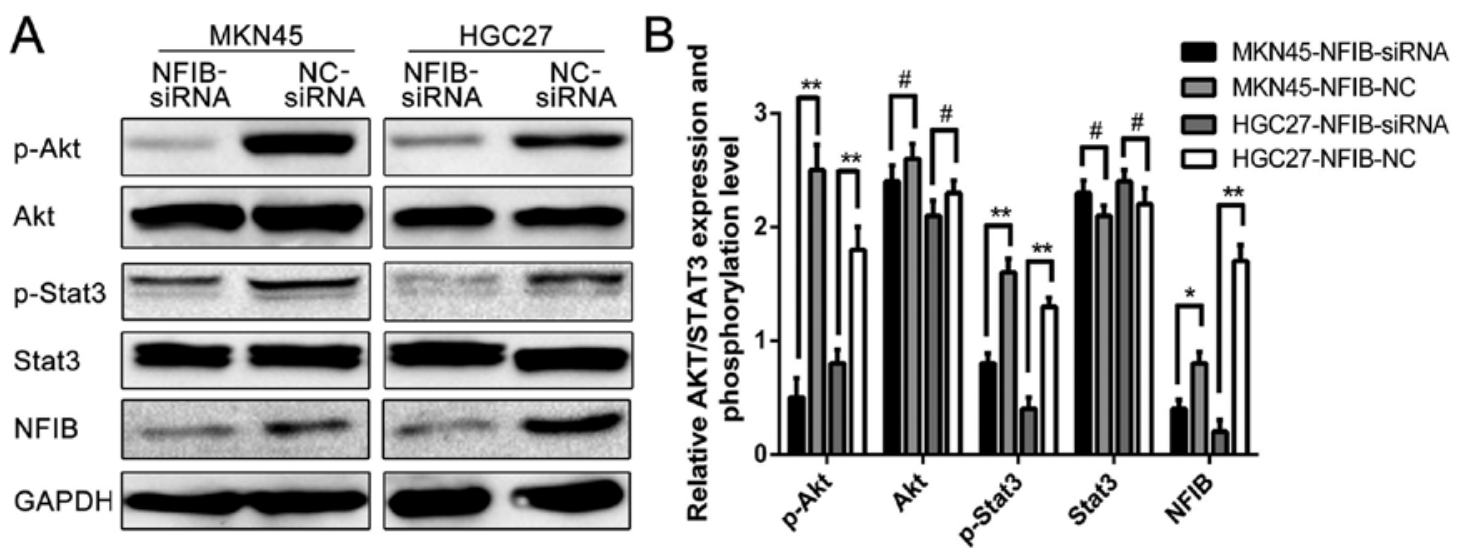

Figure 7. Western blot analysis of the phosphorylation levels of Akt and Stat3 in MKN45 and HGC27 cell lines revealed that the phosphorylation levels of all 3 proteins were significantly decreased following the transfection of NFIB-siRNA and concurrent knockdown of NFIB. No obvious difference was observed on the total protein levels of Akt and Stat $3\left({ }^{*} \mathrm{P}<0.05,{ }^{* *} \mathrm{P}<0.01,{ }^{*} \mathrm{NS}\right.$ not significant). NFIB, nuclear factor I/B.

of NFIB expression in the GC cells did in fact promote the EMT characteristics of them.

NFIB exhibits its functions in GC cells by modulating the Akt/Stat3 signaling pathways. Since we demonstrated that NFIB is an important and novel regulator for the malignancy of $\mathrm{GC}$, we were interested in determining the molecular mechanism by which NFIB functions. Since NFIB is a member of the nuclear factor family, which regulates the expression and phosphorylation of various molecules, we hypothesized that overexpressed NFIB possessed its functions in GC cells by regulating the phosphorylation of its downstream signaling molecules. A number of studies have reported that the Akt/Stat3 signaling pathway is associated with the malignancy of cancer cells, including lung (14), bladder (15) and breast cancer (16); however, no study has investigated the relationships between NFIB and the Akt/Stat3 signaling pathways yet. Thus, we explored whether NFIB affected this pathway in GC. Western blotting revealed that the expression levels of p-Akt and p-Stat 3 were suppressed when NFIB was inhibited by NFIB-siRNA, while the expression levels of total protein of these molecules exhibited no difference between the siNFIB and the siNC group (Fig. 7). The results clearly revealed that the Akt/Stat3 signaling pathway is responsible for the NFIB-regulated malignancy in GC cells, which may act as the downstream molecular signaling pathway of NFIB.

\section{Discussion}

To date, gastric cancer (GC) is still one of the most lethal and common cancers worldwide, which is often associated with a high mortality and morbidity (17-19). The majority of GC patients are diagnosed at an advanced stage and receive a poor prognosis (20). One of the reasons for the poor outcomes of GC is the malignant biological behaviors of GC cells, particularly for those with low-to-intermediate differentiation stages, which often possess strong abilities in proliferation, migration, invasion and tumorigenesis $(21,22)$.

Increasing evidence and studies have indicated that nuclear factor I/B (NFIB), a member of the nuclear factor family and a transcriptional factor necessary for the normal process of body development as well as cell division and differentiation in various types of tissues, is also critical in the formation and development of cancers (23). Previous studies have confirmed the oncogenic roles of NFIB in some types of cancer, such 
as small-cell lung cancer (24), melanoma (25) and breast cancer (26). Unlike many other classic molecules in tumor development, the roles played by NFIB are dependent of the tissue origins and the source of cancer cells. In different types of cancer, NFIB could play the part of an oncogene, a tumor suppressor, or components of gene fusion (23). However, to the best of our knowledge, the relationship between NFIB and GC has yet to be reported. Thus, the present study demonstrated for the first time that NFIB is associated to the malignancy of GC, which may be a novel aspect for the investigation of GC and a new target for the precise antitumor treatment of GC.

A recent study revealed that NFIB exerts its functions by targeting various downstream signaling pathways and molecules. For example, NFIB mediates the migration and invasion of melanoma cells through the regulation of EZH2 and MITF (25). Since NFIB belongs to a family of nuclear factors, it controls numerous cancer-related pathways, and thus, it is usually enigmatic when it comes to which pathway it controls for specific types of cancer. Finding the downstream signaling pathway for NFIB functioning in the development of $\mathrm{GC}$ is of critical importance.

It was reported that most GC patients experienced mutations in the Akt/Stat3 pathway and that this pathway could control the invasion, migration and proliferation of GC cells (27). Since Akt is a protein kinase and is responsible for the phosphorylation of different types of functional proteins, finding an association between NFIB and Akt would be helpful in explaining the oncogenic functions of NIFB in GC (28). Stat3 is another essential transcription factor that can translocate itself to the cell nucleus to regulate the expression of various pro-invasive factors, such as MMPs, HSP70 and HSP90 (29). No studies have depicted the relationship among NFIB, Akt and Stat 3 before. In the present study, we detected the interplay among the NFIB/Akt/Stat3 pathways in which NFIB served as the upstream regulator of Akt and Stat3. Notably, according to our western blot examination results, after knocking down the expression of NFIB, the phosphorylation of both Akt and Stat 3 were reduced, nevertheless, total protein expression of Akt and Stat 3 remained unchanged. This suggests that NFIB may not be connected with the Akt/Stat3 signaling pathway by directly controlling the protein expression levels of the signal molecules. Given the fact that NFIB is a nuclear factor which is responsible for the transcription of numerous nuclear proteins, which may include the activators of the Akt/Stat3 signaling pathway we thus hypothesized that NFIB, as a nuclear factor, could control the activation of the Akt/Stat 3 pathway by upregulating the expression level of its activators. Further and more extensive research is warranted to examine and ascertain our hypothesis.

Notably, our study has only initially identified the functions and roles played by NFIB in GC, and the NFIB/Akt/Stat3 signaling pathway. Our research data is not suffcient to depict the more detailed mechanisms of NFIB in the development of GC. Further investigation is warranted to determine whether NFIB contributes to other aspects of GC malignancy, such as apoptosis or the cell cycle, or if other signaling pathways are also involved in the function of NFIB in GC. Moreover, in vivo experiments are also important to be conducted in the future in order to elucidate the roles of NFIB.
In summary, we demonstrated that NFIB plays an oncogenic role in the formation, development, and metastasis of $\mathrm{GC}$, and it forms an axial relationship with Akt and Stat3 in which NFIB inhibits the phosphorylation of both Akt and Stat3. Exploring the molecular mechanism by which NFIB promotes GC malignancy through the NFIB/Akt/Stat3 axis may be helpful for the understanding of the formation and development of GC and may be the targets in GC treatment which could be insightful to develop more effective and targeted therapy for GC.

\section{Acknowledgements}

Not applicable.

\section{Funding}

The present study was supported by the The National Natural Science Foundation of China, (ID: 81600401 to C.W.) and the The Natural Science Foundation of Hubei Province, ID: 2017CFB474 to C.W.).

\section{Availability of data and materials}

The datasets used during the present study are available from the corresponding author upon reasonable request.

\section{Authors' contributions}

TR analyzed and interpreted the patient data. CW and XZ performed western blotting and functional experiments, and were major contributors in writing the manuscript. WL performed the statistical analysis of the experimental data and proofread the manuscript. WW performed the clinical data collection. KT contributed to the conception of the study and designed the research plan. All authors read and approved the manuscript and agree to be accountable for all aspects of the research in ensuring that the accuracy or integrity of any part of the work are appropriately investigated and resolved.

\section{Ethics approval and consent to participate}

The study was approved by the Ethics Committee of Union Hospital, Tongji Medical College, Huazhong University of Science and Technology. The gathering and use of the patient data and tissues were consented by all patients involved.

\section{Patient consent for publication}

Not applicable.

\section{Competing interests}

The authors declare that they have no competing interests.

\section{References}

1. Uyama I, Suda K and Satoh S: Laparoscopic surgery for advanced gastric cancer: Current status and future perspectives. J Gastric Cancer 13: 19-25, 2013. 
2. Wu C, Ruan T, Liu W, Zhu X, Pan J, Lu W, Yan C, Tao K, Zhang $\mathrm{W}$ and Zhang C: Effect and mechanism of curcumin on EZH2-miR-101 regulatory feedback loop in multiple myeloma. Curr Pharm Des 24: 564-575, 2018.

3. Abbas M, Habib M, Naveed M, Karthik K, Dhama K, Shi M and Dingding C: The relevance of gastric cancer biomarkers in prognosis and pre- and post-chemotherapy in clinical practice. Biomed Pharmacother 95: 1082-1090, 2017.

4. Li Y, Guo G, Song J, Cai Z, Yang J, Chen Z, Wang Y, Huang Y and Gao Q: B7-H3 promotes the migration and invasion of human bladder cancer cells via the PI3K/Akt/STAT3 signaling pathway. J Cancer 8: 816-824, 2017.

5. Zhang LD, Chen L, Zhang M, Qi HJ, Chen L, Chen HF, Zhong MK, Shi XJ and Li QY: Downregulation of ERR $\alpha$ inhibits angiogenesis in human umbilical vein endothelial cells through regulating VEGF production and PI3K/Akt/STAT3 signaling pathway. Eur J Pharmacol 769: 167-76, 2015.

6. Baba Y, Tamura T, Satoh Y, Gotou M, Sawada H, Ebara S, Shibuya K, Soeda J and Nakamura K: Panitumumab interaction with TAS-102 leads to combinational anticancer effects via blocking of EGFR-mediated tumor response to trifluridine. Mol Oncol 11: 1065-1077, 2017.

7. Yu B, Lv X, Su L, Li J, Yu Y, Gu Q, Yan M, Zhu Z and Liu B: MiR-148a functions as a tumor suppressor by targeting CCK-BR via inactivating STAT3 and Akt in human gastric cancer. PLoS One 11: e0158961, 2016.

8. Chen KS, Lim JWC, Richards LJ and Bunt J: The convergent roles of the nuclear factor I transcription factors in development and cancer. Cancer Lett 410: 124-138, 2017.

9. Kim J, Geyer FC, Martelotto LG, Ng CK, Lim RS, Selenica P, Li A, Pareja F, Fusco N, Edelweiss M, et al: MYBL1 rearrangements and $M Y B$ amplification in breast adenoid cystic carcinomas lacking the $M Y B-N F I B$ fusion gene. J Pathol 244: 143-150, 2018

10. Matuzelski E, Bunt J, Harkins D, Lim JWC, Gronostajski RM, Richards LJ, Harris L and Piper M: Transcriptional regulation of Nfix by NFIB drives astrocytic maturation within the developing spinal cord. Dev Biol 432: 286-297, 2017.

11. Wu Y, Zhang J, Hou S, Cheng Z and Yuan M: Non-small cell lung cancer: miR-30d suppresses tumor invasion and migration by directly targeting NFIB. Biotechnol Lett 39: 1827-1834, 2017.

12. Livak KJ and Schmittgen TD: Analysis of relative gene expression data using real-time quantitative PCR and the $2-\Delta \Delta C \mathrm{~T}$ method. Methods 25: 402-408, 2001.

13. Chen S, Zhang X, Peng J, Zhai E, He Y, Wu H, Chen C, Ma J, Wang $Z$ and Cai S: VEGF promotes gastric cancer development by upregulating CRMP4. Oncotarget 7: 17074-17086, 2016.

14. Wei YL, Dong HM, Xie ZF and Cai SX: Role of reactive oxygen species in hypoxia-induced non-small cell lung cancer migration. Zhonghua Yi Xue Za Zhi 97: 3174-3178, 2017 (In Chinese).

15. Han MH, Lee DS, Jeong JW, Hong SH, Choi IW, Cha HJ, Kim S, Kim HS, Park C, Kim GY, et al: Fucoidan induces ROS-dependent apoptosis in 5637 human bladder cancer cells by downregulating telomerase activity via inactivation of the PI3K/Akt signaling pathway. Drug Dev Res 78: 37-48, 2017.

16. Le Rhun E, Bertrand N, Dumont A, Tresch E, Le Deley MC, Mailliez A, Preusser M, Weller M, Revillion F and Bonneterre J: Identification of single nucleotide polymorphisms of the PI3K-AKT-mTOR pathway as a risk factor of central nervous system metastasis in metastatic breast cancer. Eur J Cancer 87: 189-198, 2017.
17. Ina $\mathrm{K}$ and Furuta $\mathrm{R}$ : Complete response of metastatic gastric cancer to chemoimmunotherapy. Indian J Med Res 146: 141, 2017.

18. Li Z, Wang Q, Li B, Bai B and Zhao Q: Influence of enhanced recovery after surgery programs on laparoscopy-assisted gastrectomy for gastric cancer: A systematic review and meta-analysis of randomized control trials. World J Surg Oncol 15: 207, 2017.

19. Li Q, Li H, Jiang H, Feng Y, Cui Y, Wang Y, Ji Y, Yu Y, Li W, Xu C, et al: Predictive factors of trastuzumab-based chemotherapy in HER2 positive advanced gastric cancer: A single-center prospective observational study. Clin Transl Oncol 20: 695-702, 2018.

20. Kawasaki K, Takeuchi D, Kaneko T, Miura S, Kamiya J, Miyahara Y, Yoshimura K and Ogata A: A case of advanced gastric cancer responding to neoadjuvant chemotherapy with docetaxel, cisplatin, and 5-fluorouracil, leading to a pathological complete response. Gan To Kagaku Ryoho 44: 1017-1020, 2017 (In Japanese).

21. He J, Wang X, Cai J, Wang W and Qin X: High expression of eIF3d is associated with poor prognosis in patients with gastric cancer. Cancer Manag Res 9: 539-544, 2017.

22. Sun GL, Li Z, Wang WZ, Chen Z, Zhang L, Li Q, Wei S, Li BW, $\mathrm{Xu} \mathrm{JH}$, Chen L, et al: miR-324-3p promotes gastric cancer development by activating Smad4-mediated Wnt/beta-catenin signaling pathway. J Gastroenterol, 2017.

23. Becker-Santos DD, Lonergan KM, Gronostajski RM and Lam WL: Nuclear factor I/B: A master regulator of cell differentiation with paradoxical roles in cancer. EBioMedicine 22: 2-9, 2017.

24. Dooley AL, Winslow MM, Chiang DY, Banerji S, Stransky N, Dayton TL, Snyder EL, Senna S, Whittaker CA, Bronson RT, et al: Nuclear factor I/B is an oncogene in small cell lung cancer. Genes Dev 25: 1470-1475, 2011.

25. Fane ME, Chhabra Y, Hollingsworth DE, Simmons JL, Spoerri L, Oh TG, Chauhan J, Chin T, Harris L, Harvey TJ, et al: NFIB mediates BRN2 driven melanoma cell migration and invasion through regulation of EZH2 and MITF. EBioMedicine 16: 63-75, 2017

26. Fusco N, Geyer FC, De Filippo MR, Martelotto LG, Ng CK, Piscuoglio S, Guerini-Rocco E, Schultheis AM, Fuhrmann L, Wang L, et al: Genetic events in the progression of adenoid cystic carcinoma of the breast to high-grade triple-negative breast cancer. Mod Pathol 29: 1292-1305, 2016.

27. Yoshida R, Sasaki T, Minami Y, Hibino Y, Okumura S, Sado M, Miyokawa N, Hayashi S, Kitada M and Ohsaki Y: Activation of Src signaling mediates acquired resistance to ALK inhibition in lung cancer. Int J Oncol 51: 1533-1540, 2017.

28. Mo D, Fang H, Niu K, Liu J, Wu M, Li S, Zhu T, Aleskandarany MA, Arora A, Lobo DN, et al: Human helicase RECQL4 drives cisplatin resistance in gastric cancer by activating an AKT-YB1-MDR1 signaling pathway. Cancer Res 76: 3057-66, 2016.

29. Ma DH, Li BS, Liu JJ, Xiao YF, Yong X, Wang SM, Wu YY, Zhu HB, Wang DX and Yang SM: miR-93-5p/IFNAR1 axis promotes gastric cancer metastasis through activating the STAT3 signaling pathway. Cancer Lett 408: 23-32, 2017. 